\title{
Endoluminal tumor implant of a colorectal cancer in an anal fistula detected by FISH techniques: a case report
}

\author{
Enrique Montero-Mateos ${ }^{1}$, Sofía del Carmen ${ }^{1}$, Julia Sanz ${ }^{1}$, Raquel Rodriguez-García ${ }^{2}$, \\ José Antonio Alcázar ${ }^{2}$, José María Sayagués ${ }^{1}$, Mar Abad $^{1}$ \\ ${ }^{1}$ Servicio de Anatomía Patológica, Hospital Clínico Universitario de Salamanca e Instituto de Investigación Biomédica de Salamanca (IBSAL), \\ Universidad de Salamanca, Salamanca, Spain; ${ }^{2}$ Servicio de Cirugía General y del Aparato Digestivo, Hospital Clínico Universitario de Salamanca e \\ Instituto de Investigación Biomédica de Salamanca (IBSAL), Universidad de Salamanca, Salamanca, Spain \\ Correspondence to: Mar Abad; José María Sayagués. Servicio de Anatomía Patológica. Paseo San Vicente S/N. 37007 Salamanca, Spain. Email: \\ marabad@usal.es; Email: ppmari@usal.es.
}

\begin{abstract}
Intraluminal shedding of tumor cells is a rare infrequent sporadic colorectal cancer (sCRC) mechanism of spreading. Less than 30 cases of sCRC metastasis into anal fistula have been reported. Here, we study a 72-year-old male with an adenocarcinoma arising in an anal fistula. Subsequent studies revealed another tumor in the rectum without distant metastatic disease; therefore, a curative-intent abdominoperineal resection was performed. The histologic study showed a moderately differentiated adenocarcinoma in both locations. No perineural or lymphovascular invasion was observed, and all the lymphatic nodes resected were negative for malignancy. Both tumors showed positive CK20 and negative CK7 immunostaining, but KRAS G12D mutation was only detected in the rectal tumor. After those conventional studies, a cytogenetic profile of both tumors was performed by interphase fluorescence in situ hybridization (iFISH) techniques. The FISH study displayed an identical genetic profile in both tumors, loss of the chromosomes 8 and 18q, and no alteration in chromosome 7 and 13q. Based on pathological and genetic findings, we established the same clonal origin of both tumors. Currently, the diagnosis of an intraluminal CRC metastasis relies on histologic and immunohistochemistry findings. We suggest that genetic studies at the individual cell level by FISH techniques may be useful in order to differentiate synchronous from intraluminal metastasis.
\end{abstract}

Keywords: Sporadic colorectal cancer (sCRC); endoluminal tumor implant; genetic abnormalities; fluorescence in situ hybridization (FISH)

Submitted Jul 22, 2020. Accepted for publication Nov 17, 2020.

doi: 10.21037/jgo-20-281

View this article at: http://dx.doi.org/10.21037/jgo-20-281

\section{Introduction}

Sporadic colorectal cancer (sCRC) represents the second leading cause of cancer-related mortality in Europe, being responsible for 177,400 deaths in 2018 (1). Metastatic dissemination of the primary tumor is the major cause of death among sCRC patients. The most common locations of sCRC metastasis are liver, lung and peritoneum. Hematogenous and lymphatic spreading is a well-known mechanism of dissemination. Much less infrequent is the intraluminal shedding with distant intraluminal metastasis. In these cases, the genetic characterization of each of the tumor cell clones present in the primary tumor and in the metastases could help to establish the clonal relationship between them both. In this sense, interphase fluorescence in situ hybridization (iFISH) techniques, although it does not provide specific information about each chromosomal abnormality present in a tumor, with a suitable combination of probes, is used to obtain information about the genetic diversity of a tumor at the single-cell level.

Here, we report a case of sCRC that metastasized in an anal fistula and how a detailed analysis of the chromosomal abnormalities detected by iFISH in the different tumor cell clones identified in each tumor sample (primary and in 


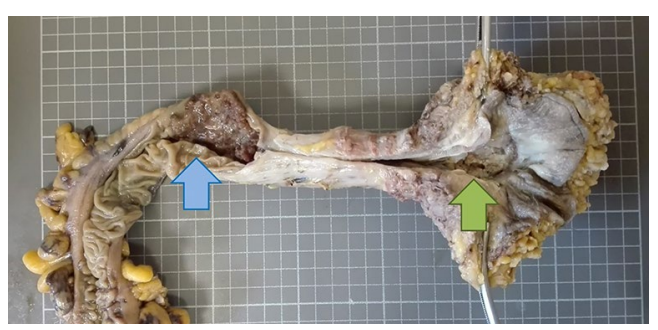

Figure 1 Surgical piece of abdominoperineal amputation in which two tumors are observed, a rectal (blue arrow), and one in the anal margin (green arrow).

anal fistula tumor) allowed us to determine the same clonal origin between both tumors

We present the following case in accordance with the CARE reporting checklist (Available at http://dx.doi. org/10.21037/jgo-20-281).

\section{Case presentation}

A 72-year-old man consulted for perineal pain and was initially diagnosed with anal fistula. A partial fistulotomy and cutting seton technique was performed in an outpatient setting, and biopsies were remitted to the pathology department. The histology study revealed a moderately differentiated adenocarcinoma; therefore, the patient was admitted completing the extension study. The colonoscopy showed a second tumor at $15 \mathrm{~cm}$ of the anal margin. The CT-scan did not show any distant metastases, peritoneal involvement, or abdominal suspicious lymph nodes. At this point, curative-intent surgery was offered and accepted. Abdominoperineal resection was performed and remitted to the Pathology department. The macroscopic study (Figure 1) displayed a 6-cm rectal tumor and another 3-cm tumor at the anal margin. The histologic study showed a moderately differentiated adenocarcinoma in both locations. The rectal tumor showed extension into pericolorectal fat; meanwhile, the anal tumor demonstrated invasion of the sphincter muscle. No perineural or lymphovascular invasion was observed, and all the lymphatic nodes resected were negative for malignancy. Furthermore, both tumors showed positive CK20 and negative CK7 immunostaining (Figure 2).

After conventional histological studies, a cytogenetic profile of both tumors was performed. For this, a set of 7 different probes specific for those chromosomes and chromosomal regions most frequently gained/amplified and

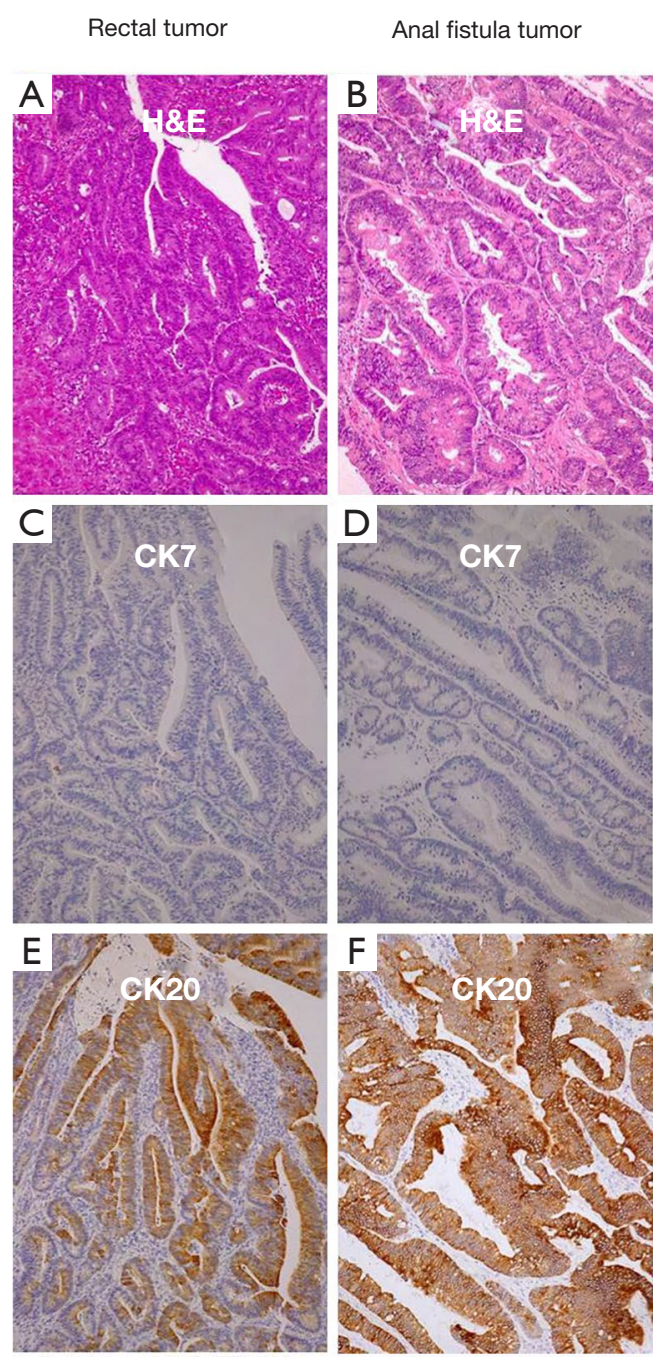

Figure 2 Comparison between the immunophenotypic features of the rectal tumor and the anal fistula tumor $(20 x)$. Rectal tumor; hematoxylin-eosin (A), cytokeratin 7 (C) and cytokeratin $20(\mathrm{E})$. Anal fistula tumor; hematoxylin-eosin (B), cytokeratin 7 (D) and cytokeratin 20 (F). Both adenocarcinomas showed a CK7-/CK20+ staining pattern.

deleted in sCRC (2) were analyzed by iFISH techniques: $7 \mathrm{q} 31$ (D7S486), 7p11.1 (centromere), 8p22 (LPL), 8p11.1 (centromere) and 8q24 (CMYC), 13q14 (FOXO1) and 18q21 (BCL2); Vysis Inc, Chicago, IL, USA. FISH study displayed an identical genetic profile in both tumors, loss of the chromosomes 8 and 18q, and no alteration in chromosome 7 and $13 \mathrm{q}$; consequently, a common clonal origin was suggested (Figure 3).

Eventually, KRAS, NRAS and BRAF genes were studied using PCR techniques and also mismatch repair genes 

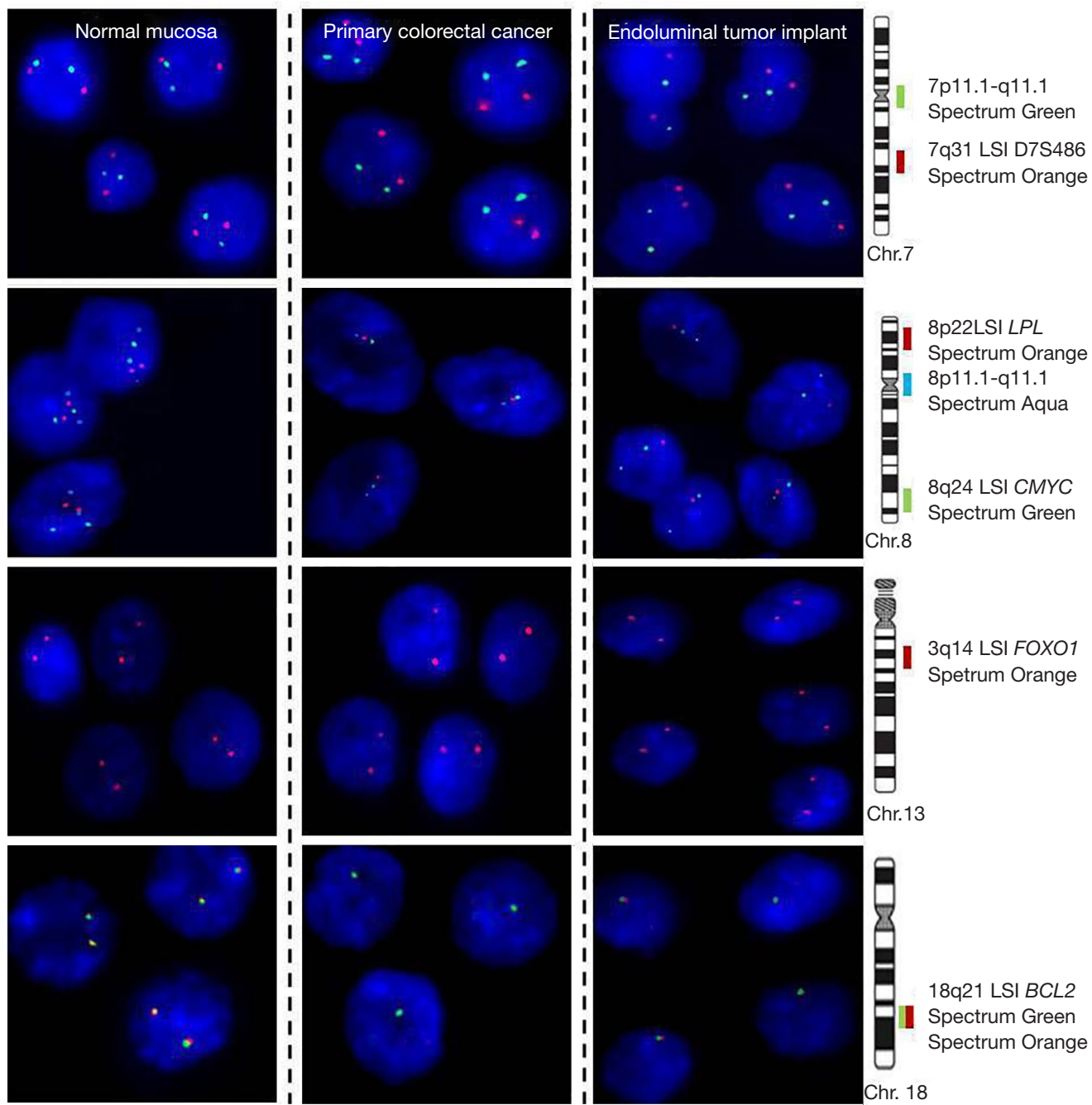

Figure 3 Representative pictures of cell nuclei from primary colorectal cancer, endoluminal implant and normal mucosa after hybridization with two probes for chromosome 7 (7p11.1 and 7q31; green and red signals, respectively), three probes for chromosome 8 (8p22, 8p11, and 8q24; red, aqua and green signals, respectively), one probe for chromosome 13q (13q14; red signals) and two probes (break apart probes) for chromosome 18q (18q21; green and red signals). All cell nuclei of both tumors showed a normal diploid number of hybridization signals for the probes of the chromosome 7 and $13 \mathrm{q}$ and loss of a whole chromosome 8 and of the 18q21 chromosomal region, suggesting a common clonal origin.

(MMR; MLH1, MSH2, MSH6 and PMS2) were assessed by immunochemistry (IHC). NRAS, BRAF and MMR genes showed no mutation, whereas KRAS G12D (c.35G>A; p.Gly12Asp) mutation was only detected in the rectal tumor.

All procedures performed in studies involving human participants were in accordance with the ethical standards of the institutional and/or national research committee(s) and with the Helsinki Declaration (as revised in 2013). The study was reviewed and approved by the ethics committee of the University Hospital of Salamanca. The patient referred in the case report participant provided informed written consent.

\section{Discussion}

Intraluminal metastases of sCRC are unusual. The mechanisms underlying in the shedding of exfoliated tumor 
cells is not well understood, but it has been demonstrated experimentally that sCRC sheds viable cells capable of implanting endoluminally (3). By this mechanism, several endoluminal sCRC metastases have been suggested in different locations: colorectal anastomosis, colic ulcers, hemorrhoids, small bowel or common bile ducts (3-6). Anal metastasis has been estimated at $0.05 \%$ (95\% CI, $0.006-$ $0.08 \%$ ) of patients with sCRC, and the metastatic mechanism was considered an intraluminal spread in about $80 \%$ of cases (7). Most of the intraluminal anal metastasis reported arise in a preexisting lesion: about two-thirds implanted into anal fistula and one fifth in iatrogenic scarring (7). Less than 30 cases of sCRC metastasis into anal fistula have been reported and they may be the first symptom of a left-sided sCRC, most frequently in patients with an advanced clinical stage (7-10). Since primary sCRC and anal metastasis are usually discovered at the same time, various attempts to differentiate synchronous from a metastatic anal tumor have been done, based on several criteria: similar histological findings, lack of dysplasia in the surrounding anal glands, lack of mucin and the absence of lymph node and vasculolymphatic invasion (11). Furthermore, a concordant immunochemistry cytokeratin 7 negative and cytokeratin 20 positive supports a common origin and helps rule out an anal gland adenocarcinoma. In general, the patients reported were treated with curative-intent surgery (in some cases with chemotherapy or chemoradiotherapy associated) and showed good prognosis, better than in cases with lymphatic or hematogenous spread (11).

Several studies have explored the cytogenetic alterations in sCRC, showing that gains/amplifications of chromosomes $7 \mathrm{p}, 8 \mathrm{q}, 13 \mathrm{q}$ and $20 \mathrm{q}$ together with losses of the $1 \mathrm{p}, 8 \mathrm{p}$, and $17 \mathrm{p}$ chromosomal regions are frequent findings in these tumors (12). Although the metastatic tumor usually harbors more chromosomic abnormalities that of their paired primary tumor, it is well known that share highly similar karyotypic abnormalities. Many of these chromosomic abnormalities are acquired before the tumoral spreading $(13,14)$. In our case, iFISH studies revealed that both tumors displayed loss of the chromosomes 8 and $18 \mathrm{q}$ without alteration of the chromosomes 7 and $13 \mathrm{q}$. The former is an unusual finding, as Duijf et al. described loss of chromosome 8 in $0.96 \%$ of 520 sCRC tumors (15). Conversely, chromosomes 7 and $13 \mathrm{q}$ gains have been described in $30 \%$ in nonmetastatic disease and are significantly more frequent in metastatic tumors $(\mathrm{P}<0.05$; $61 \%$ and $75 \%$, respectively) (16). Those shared infrequent cytogenetic alterations among both tumors support the existence of a close genetic relationship between primary tumors and their intraluminal metastasis, suggesting a common clonal origin instead of synchronous sCRC. Given that some authors propose a more aggressive approach for synchronous sCRC-suggesting subtotal or total colectomy in that cases-differentiate between synchronous and metastatic tumors is important to choose the most adequate therapeutic option $(17,18)$. The generalized use of iFISH techniques in pathology departments makes it a useful and accessible option in daily clinical practice to make an accurate diagnosis in these patients.

Conventional cytogenetics or Spectral Karyotyping (SKY) to define the chromosomal and molecular abnormalities of sCRC has important limitations, mainly related to the need for obtaining tumor metaphases, after an in vitro cell culture period (2); even more, when normal metaphases are obtained it is difficult to confirm that they correspond to the tumor cells and not to the normal cells present in the sample. These include the potential existence of clonal selection during cell culture, the analysis of a small fraction (typically $\leq 1 \%$ ) of all the cells present in the tumor sample, and the difficulties in obtaining tumor cell metaphases in a significant proportion of cases. In addition, other molecular approaches such as array comparative genomic hybridization (aCGH) or single nucleotide polymorphism arrays (aSNPs) also has several limitations: the detection of gains or losses of genetic material present in the majority (e.g., more than 50\%) of cells in the tumor sample from which DNA was extracted (12). These approaches do not provide detailed information about the clonal heterogeneity and genetic diversity of a given tumor and the potential co-existence of different abnormalities in the same tumor sample, particularly when tumor cell clones are present at relatively low frequencies (2). Conversely, iFISH techniques, with an adequate combination of probes, we can to obtain information about the genetic heterogeneity of a tumor at the single-cell level.

On the other hand, the mutation KRAS discordance observed between the primary tumor and the endoluminal implant may be explained by two mechanisms. The first hypothesis explains that it is a consequence of the divergent evolution of the tumors once the metastases are established. The presence of WT KRAS-metastasis with KRAS-mutated primary tumor has been reported in 3 of 99 cases (3\%) by Santini et al. (19). and in 5 cases of 305 patients (2\%) by Knijn et al. (20). Furthermore, Miranda et al. described a lower rate of mutated $K R A S$ in lymph node metastasis than in primary tumor or visceral metastasis, 
suggesting that those colonizing neoplastic cells spread through lymphatics before KRAS mutation occurs (21). They hypothesize that native $K R A S$ confers an advantage in the ability to migrate to lymph nodes. A similar mechanism may underlie in intraluminal metastasis; nevertheless, the data available is scant, and further research is necessary. The second possibility is that because of the primary tumor heterogeneity (a well-known circumstance in sCRC), tissue sampling has not included KRAS-mutated areas (22).

\section{Conclusions}

In conclusion, the use of an accessible technique as iFISH may be an additional useful instrument for the assessment of anal tumors in order to establish their metastatic nature if there is a CRC coexisting tumor.

\section{Acknowledgments}

Funding: This work has been partially supported by grants from Gerencia Regional de Salud de Castilla y León, Valladolid, Spain (GRS1302/A/16) and GRS2051/ A/19), Ministerio de Ciencia e Innovación (PID2020118703RB-I00), and Fundación Memoria de Don Samuel Solórzano Barruso, Universidad de Salamanca, Salamanca, Spain.

\section{Footnote}

Reporting Checklist: The authors have completed the CARE reporting checklist. Available at http://dx.doi.org/10.21037/ jgo-20-281

Peer Review File: Available at http://dx.doi.org/10.21037/ jgo-20-281

Conflicts of Interest: All authors have completed the ICMJE uniform disclosure form (available at http://dx.doi. org/10.21037/jgo-20-281). The authors have no conflicts of interest to declare.

Ethical Statement: The authors are accountable for all aspects of the work in ensuring that questions related to the accuracy or integrity of any part of the work are appropriately investigated and resolved. All procedures performed in studies involving human participants were in accordance with the ethical standards of the institutional and/or national research committee(s) and with the Helsinki
Declaration (as revised in 2013). The study was reviewed and approved by the ethics committee of the University Hospital of Salamanca. The patient referred in the case report participant provided informed written consent

Open Access Statement: This is an Open Access article distributed in accordance with the Creative Commons Attribution-NonCommercial-NoDerivs 4.0 International License (CC BY-NC-ND 4.0), which permits the noncommercial replication and distribution of the article with the strict proviso that no changes or edits are made and the original work is properly cited (including links to both the formal publication through the relevant DOI and the license). See: https://creativecommons.org/licenses/by-nc-nd/4.0/.

\section{References}

1. Malvezzi M, Carioli G, Bertuccio P, et al. European cancer mortality predictions for the year 2018 with focus on colorectal cancer. Ann Oncol 2018;29:1016-22.

2. Sayagués JM, Abad MM, Melchor HB, et al. Intratumoural cytogenetic heterogeneity of sporadic colorectal carcinomas suggests several pathways to liver metastasis. J Pathol 2010;221:308-19.

3. Matsuda A, Kishi T, Musso G, et al. The effect of intraoperative rectal washout on local recurrence after rectal cancer surgery: A meta-analysis. Ann Surg Oncol 2013;20:856-63.

4. Ishiyama S, Inoue S, Kobayashi K, et al. Implantation of rectal cancer in an anal fistula: Report of a case. Surg Today 2006;36:747-9.

5. Soh TIP, Doshi BK, Putti TC, et al. Common bile duct obstruction by intraluminal colorectal metastasis without evidence of liver or intraabdominal nodal metastasis. Int $\mathrm{J}$ Colorectal Dis 2012;27:127-8.

6. Stamopoulos P, Machairas N, Kykalos S, et al. Intraluminal rectal cancer metastasis to the small bowel: An extremely rare case report. Mol Clin Oncol 2017;7:553-6.

7. Takahashi H, Ikeda M, Takemasa I, et al. Anal metastasis of colorectal carcinoma origin: Implications for diagnosis and treatment strategy. Dis Colon Rectum 2011;54:472-81.

8. Benjelloun el B, Aitalalim S, Chbani L, et al. Rectosigmoid adenocarcinoma revealed by metastatic anal fistula. The visible part of the iceberg: a report of two cases with literature review. World J Surg Oncol 2012;10:209.

9. Sasaki S, Sugiyama M, Nakaji $Y$, et al. Anal metastasis of rectal cancer-adenocarcinoma of squamous cells: a case report and literature review. Surg Case Rep 2017;3:55. 
10. Gupta R, Kay M, Birch DW. Implantation metastasis from adenocarcinoma of the colon into a fistula-in-ano: A case report. Can J Surg 2005;48:162-3.

11. Ikeda T, Nanashima A, Ichihara A, et al. A rare case of rectal cancer with perianal metastasis: A case report. World J Surg Oncol 2019;17:149.

12. Sayagués JM, Fontanillo C, Abad Mdel M, et al. Mapping of genetic abnormalities of primary tumours from metastatic CRC by high-resolution SNP arrays. PLoS One 2010;5:e13752.

13. Bardi G, Parada LA, Bomme L, et al. Cytogenetic findings in metastases from colorectal cancer. Int J Cancer 1997;72:604-7.

14. Sylvester BE, Vakiani E. Tumor evolution and intratumor heterogeneity in colorectal carcinoma: Insights from comparative genomic profiling of primary tumors and matched metastases. J Gastrointest Oncol 2015;6:668-75.

15. Duijf PHG, Schultz N, Benezra R. Cancer cells preferentially lose small chromosomes. Int J cancer 2013;132:2316-26.

16. González-González M, Fontanillo C, Abad MM, et al. Identification of a characteristic copy number alteration profile by high-resolution single nucleotide polymorphism arrays associated with metastatic sporadic colorectal

Cite this article as: Montero-Mateos E, del Carmen S, Sanz J, Rodriguez-García R, Alcázar JA, Sayagués JM, Abad M. Endoluminal tumor implant of a colorectal cancer in an anal fistula detected by FISH techniques: a case report. J Gastrointest Oncol 2021;12(2):900-905. doi: 10.21037/jgo-20-281 cancer. Cancer 2014;120:1948-59.

17. Lee BC, Yu CS, Kim J, et al. Clinicopathological features and surgical options for synchronous colorectal cancer. Medicine (Baltimore) 2017;96:e6224.

18. Yang J, Peng JY, Chen W. Synchronous colorectal cancers: A review of clinical features, diagnosis, treatment, and prognosis. Dig Surg 2011;28:379-85.

19. Santini D, Loupakis F, Vincenzi B, et al. High concordance of KRAS status between primary colorectal tumors and related metastatic sites: implications for clinical practice. Oncologist 2008;13:1270-5.

20. Knijn N, Mekenkamp LJM, Klomp M, et al. KRAS mutation analysis: A comparison between primary tumours and matched liver metastases in 305 colorectal cancer patients. Br J Cancer 2011;104:1020-6.

21. Miranda E, Bianchi P, Destro A, et al. Genetic and epigenetic alterations in primary colorectal cancers and related lymph node and liver metastases. Cancer 2013;119:266-76.

22. Del Carmen S, Sayagués JM, Bengoechea O, et al. Spatio-temporal tumor heterogeneity in metastatic CRC tumors: A mutational-based approach. Oncotarget 2018;9:34279-88. 